\title{
Improving time to antibiotics and implementing the "Sepsis 6"
}

\author{
Calum McGregor
}

NHS Lanarkshire, UK

\begin{abstract}
It has been shown that completion of the "Sepsis 6" within 1 hour reduces mortality (1). This project aims to assess compliance with this standard and evaluate the effectiveness of a sepsis improvement plan in a district general hospital in the UK.
\end{abstract}

\begin{abstract}
A baseline audit was performed, examining case notes of "septic patients" retrospectively (those on intravenous antibiotics). Compliance with each element of the sepsis six plus time to first antibiotic (TTFA) was assessed. A sepsis improvement plan was introduced consisting of staff education, reinforcing vigilance, regular multidisciplinary meetings and incorporating a standardised approach through the use of a sepsis proforma. Following the introduction of this, and after some refinement, the average time to antibiotic fell from 6 hours to 1.4 hours.
\end{abstract}

In conclusion, an educational drive along with a systematic change in processes has seen reduced TTFA along with enhanced compliance with most elements of the sepsis 6 . Through continued assessment and further improving upon systematic processes with continued education we would anticipate consistent improvement in the management of septic patients.

\section{Problem}

Left untreated, sepsis can progress to severe sepsis and septic shock, having a mortality rate of $30 \%$ and $50 \%$ respectively (2). Early recognition and resuscitation with appropriate antibiotic therapy is therefore essential to optimise survival rates. The "sepsis $6 "$ are 6 processes which, when done within an hour, have been shown to improve mortality (1). A baseline audit showed that we were not completing the sepsis 6 within an hour in septic patients. This was potentially leading to delays in treatment for septic patients in the emergency department (ED), and the medical receiving unit, of our district general hospital in Lanarkshire, UK.

A number of sources of delays were found - delays to first assessment, delays from assessment to review by medical staff, delays from being seen by medical staff to prescription of antibiotics and delays from prescription to administration of antibiotics. System issues contributed to some of these delays - for example a lack of a robust triage for septic patients; and logistical issues - for example, the antibiotics given as part of our neutropenic sepsis protocol were located in a separate ward from our ED and receiving unit. There has also been an almost $50 \%$ increase in medical admissions to our hospital over the past 10 years, making it more difficult for junior doctors to see patients promptly. In addition, sepsis can be difficult to recognise, with $87 \%$ of physicians stating that, "symptoms of sepsis can easily be attributed to other conditions, causing late or mis-diagnosis"(3), which may have been contributing to the delays.

\section{Background}

For patients with septic shock, there is an $8 \%$ increase in mortality for every hour of delay in antibiotic administration (4).

Completion of the sepsis 6 within one hour has been shown to improve mortality (1). The sepsis 6 is an example of a care bundle. The Institute for Healthcare Improvement state that a care bundle is:

"A structured way of improving processes of care and patient outcomes. It is a small straightforward set of practices - generally three to five - that, when performed collectively, reliably and continuously, have been proven to improve patient outcomes."

The components of the sepsis 6 are: blood cultures, check full blood count and lactate, IV fluid challenge, IV antibiotics, monitor urine output and give oxygen. Patients with a lactate over 4 due to sepsis have a mortality of approximately $40 \%$ (5), therefore knowing the lactate helps to escalate appropriate patients early (6). Obtaining blood cultures prior to prompt administration of antibiotics is associated with a reduction in mortality for septic patients (7).

Completing the sepsis 6 is associated with a reduction in mortality, in comparison with those who did not receive the sepsis 6 within an hour (1). In addition, completing the Surviving Sepsis Campaign's resuscitation bundle, which includes early administration of antibiotics, is associated with a reduction in mortality (5). In summary, introducing a care bundle in the form of the sepsis 6 has been shown to improve mortality in a number of settings.

\section{Baseline Measurement}

Our baseline measurement is shown in the attachment below, and our average time to antibiotic at baseline was six hours. We measured time taken to complete each component of the sepsis 6 , and whether or not each component was achieved within an hour of identification of the septic patient. Patients were identified by retrospective case note review of patients commenced on IV antibiotics, and were included if they fulfilled the criteria of MEWS of 4 or greater, SIRS of 2 or greater and suspicion of new infection. 
A sepsis group was subsequently set up, and after consultation with the national literature and the Scottish Patient Safety Programme, patients to be included in the audit were those with a Modified Early Warning Score (MEWS) of 4, Systemic Inflammatory Response Syndrome (SIRS) score of 2 and suspicion of new infection. The definitions used for SIRS scoring were as follows:

Temperature $<36$ or $>38$

Respiratory rate $>20$

Heart Rate $>90$

White Cell Count $<4$ or $>12$

See supplementary file: ds2450.doc - "Baseline Audit Data"

\section{Design}

A number of factors were identified as contributing to delays in completing the Sepsis 6 within an hour. A multi-factorial approach was therefore introduced. Education sessions involving triage nurses, staff nurses, clinical support workers and junior medical staff were introduced, along with educational sessions for the labs, to alert them to the possibility of increased numbers of lactates and blood cultures being performed. In addition, a sepsis integrated care pathway was introduced, with clear instructions on what should be done within an hour, and poster reminders, containing a flowchart of how to manage the septic patient, were introduced.

Data was collected and fed back to the staff in the emergency department, and receiving unit, which had a very encouraging and positive effect on staff engagement with the process. Other innovations were introduced, such as a 1 hour stop-clock which is triggered when a septic patient is identified. A sepsis group involving a multi-disciplinary team from the two areas involved, met on a weekly basis to discuss how to improve the management of sepsis further, and this is ongoing.

\section{Strategy}

PDSA 1 - Following a multi-disciplinary educational campaign, including inviting the sepsis clinical lead for Scotland to give a lecture, a sepsis integrated care pathway was introduced. Data started to be collected, and was then analysed to identify unnecessary delays.

PDSA 2 - It was identified that delays were occurring as the antibiotic used in our neutropenic sepsis policy was not kept in the Emergency Department. This was rectified and the time to antibiotic administration improved.

PDSA 3 - Data analysis showed a delay between identification of the septic patient and being seen by a junior doctor. In order to improve this, a more robust triage system was introduced, with septic patients being triaged with a higher priority category than previously. One hour stop-clocks were also introduced, to re- enforce the importance of prompt treatment.

PDSA 4 - Simulation and real-time observation showed that different equipment required for the Sepsis 6 - ie. blood culture bottles, IV cannulas and blood containers for lactate, were located in different sections of the ward, therefore we introduced a sepsis trolley to centralise the equipment and avoid delays.

PDSA 5 - The wording on the ICP was changed, and in order to avoid inappropriate catheterisation and oxygen therapy, a Not Applicable column was introduced. However, this led to confusion as it could then be interpreted that all aspects of the sepsis 6 (including antibiotics) could be not-applicable. The wording of the ICP therefore had to be changed again, without a not applicable column, but with clearer instructions on when giving oxygen and catheterising patients were indicated.

\section{Results}

After completion of the project, our repeat audit data is attached below, and the median time to antibiotic was reduced to 1.4 hours. Patients were entered into the project if they met the following criteria: MEWS score of 4 or greater, SIRS score of 2 or greater and suspicion of new infection. This improvement has been sustained over the subsequent months, as shown by the graphs attached below.

See supplementary file: ds2455.doc - "Median Time to Antibiotic and compliance with the sepsis six"

\section{Lessons and Limitations}

Introducing a care bundle can be effective in improving the initial management of septic patients.

Analysing each component of the bundle helps to reduce delays. Involving a multi-disciplinary team will help to sustain improvements in the long term. The PDSA cycle is useful in implementing small scale changes which can have a significant effect.

Limitations are that educational drive alone will not lead to the maximum improvement. Achieving the sepsis 6 within an hour in a busy district general reliably is challenging. The method used for data collection in this study could potentially have resulted in some patients who fulfilled the criteria for sepsis being missed, as if they were not recorded in the sepsis book they would not be included in the results. There were a number of challenges with this project. One challenge was keeping track of patients involved in the study, in order to collect the data. To facilitate this, we developed a sepsis book, which had a tear off strip at the bottom of the ICP, meaning that we had a record of all the patients included on the ICP. Another challenge was overcoming some initial concern from the laboratory staff, as they were worried about the additional potential workload for them. However, through education and raising there awareness of the sepsis campaign, the lab staff were very supportive and helpful. 


\section{BMJ Quality Improvement Reports}

We would hope that the results will continue to improve through regular review and refinement of the processes used.

Improvements seen in this project have only been achievable through the involvement of a wide variety of staff. Given the staff engagement with the process, and the positive reinforcement of seeing ongoing improvements in their performance, we are confident that the improvements will be sustainable in the long term.

\section{Conclusion}

Introduction of a care bundle, along with an educational drive, has resulted in a significant improvement in the initial management of septic patients in our hospital.

\section{References}

1. Daniels et al. The Sepsis Six and the Severe Sepsis Resuscitation Bundle: A Prospective Observational Cohort Study. Emergency Medicine Journal. 2011;( 28):507-512.

2. Bernard GR et al. Efficacy and safety of recombinant human activated protein $\mathrm{C}$ for severe sepsis. New England Journal of Medicine. 2001;(344): 699-709.

3. Poeze $M$ et al. An international sepsis survey: a study of doctors' knowledge and perception about sepsis. Critical Care. 2004;(8):R409-R413

4. Kumar A et al.Duration of hypotension prior to initiation of effective antimicrobial therapy is the critical determinant of survival in human septic shock. Critical Care Medicine. 2006;(34):1589-1596.

5. Daniels, R. Surviving the first hours in sepsis: getting the basics right. Journal of Antimicrobial Chemotherapy. 2011;(66(s2)): ii11-ii13.

6. Drumheller B, Goyal M, Pines J et al. Elevated point-of-care lactate at triage is predictive of admission among sepsis patients presenting to the emergency department. Annals of Emergency Medicine. 2007;(50):S21-2.

7. Levy MM et al. The Surviving Sepsis Campaign: results of an international guideline-based performance improvement program targeting severe sepsis. Critical Care Medicine 2010;(38):1-10.

\section{Declaration of interests}

Nothing to declare.

\section{Acknowledgements}

Dr Robert Hart, Dr Chris Lochrin, Dr Mhairi Sinclair, Martin

Carberry, Tracy Dodd and Dr Sanjiv Chohan. 